\title{
Experimentally verified pulse formation model for high-power femtosecond VECSELs
}

\author{
Oliver D. Sieber • Martin Hoffmann • Valentin J. Wittwer • \\ Mario Mangold $\cdot$ Matthias Golling $\cdot$ Bauke W. Tilma • \\ Thomas Südmeyer $\cdot$ Ursula Keller
}

Received: 16 February 2013/Accepted: 11 April 2013/Published online: 27 April 2013

(C) The Author(s) 2013. This article is published with open access at Springerlink.com

\begin{abstract}
Optically pumped vertical-external-cavity surface-emitting lasers (OP-VECSELs), passively modelocked with a semiconductor saturable absorber mirror (SESAM), have generated the highest average output power from any sub-picosecond semiconductor laser. Many applications, including frequency comb synthesis and coherent supercontinuum generation, require pulses in the sub-300-fs regime. A quantitative understanding of the pulse formation mechanism is required in order to reach this regime while maintaining stable, high-average-power performance. We present a numerical model with which we have obtained excellent quantitative agreement with two recent experiments in the femtosecond regime, and we have been able to correctly predict both the observed pulse duration and the output power for the first time. Our numerical model not only confirms the soliton-like pulse formation in the femtosecond regime, but also allows us to develop several clear guidelines to scale the performance toward shorter pulses and higher average output power. In particular, we show that a key VECSEL design parameter is a high gain saturation fluence. By optimizing this parameter, 200-fs pulses with an average output power of more than $1 \mathrm{~W}$ should be possible.
\end{abstract}

\section{Introduction}

Vertical-external-cavity surface-emitting lasers (VECSELs) [1] passively modelocked [2] with an intra-cavity

O. D. Sieber $(\bowtie) \cdot$ M. Hoffmann · V. J. Wittwer .

M. Mangold · M. Golling · B. W. Tilma - T. Südmeyer ·

U. Keller

Department of Physics, Institute for Quantum Electronics, ETH

Zürich, Wolfgang-Pauli-Str. 16, 8093 Zurich, Switzerland

e-mail: siebero@phys.ethz.ch semiconductor saturable absorber mirror (SESAM) [3-5] and Modelocked Integrated eXternal-cavity SurfaceEmitting Lasers (MIXSEL) [6, 7] benefit from the advantages of diode-pumped solid-state lasers (DPSSL), such as excellent beam quality and high-average output power at high repetition rates [2] and do not suffer from Q-switching instabilities [8]. The semiconductor gain chip enables bandgap engineering to provide a high degree of flexibility in the operation wavelength [9]. Modelocked VECSELs with high-Q cavities have been demonstrated, resulting in a low timing jitter noise $[10,11]$, which is comparable to the noise performance of ion-doped solid-state lasers [12]. This makes them very interesting for optical communication [13] and frequency metrology applications [14, 15]. The possibility of developing high-average power frequency comb sources at $\mathrm{GHz}$ repetition rates enables a high power per comb line and an easier access to the individual comb lines. A frequency comb from a modelocked laser is typically stabilized with an f-to- $2 \mathrm{f}$ interferometer [16] to detect and stabilize the frequency comb offset (i.e., carrierenvelope offset (CEO) frequency) and a simple cavity length adjustment to stabilize the frequency comb spacing (i.e., pulse repetition rate) [17]. For a self-referenced f-to$2 \mathrm{f}$ stabilization, a coherent octave-spanning supercontinuum is required which is typically generated in a nonlinear fiber. Work is in progress to relax the laser parameters for coherent supercontinuum generation. For example, in Ref. [14], we demonstrated stable frequency comb generation starting from up to $\approx 200$ fs pulses from a diode-pumped Er:Yb:glass laser passively modelocked with a SESAM. Compared to fiber frequency combs, we obtained much better noise performance [15, 18]. More recently, we extended this work to gigahertz Yb-doped DPSSLs with femtosecond pulses at multi-watt average output power $[19,20]$. 
So far, the best performance of picosecond and femtosecond VECSELs has been achieved with SESAM modelocking [2]. The SESAM can be integrated into the VECSEL structure, such that the gain layers and the saturable absorber layers are integrated into one single semiconductor chip, referred to as the MIXSEL structure [6]. To date, the highest average output power from a passively modelocked semiconductor laser has been obtained with such a MIXSEL generating up to $6.4 \mathrm{~W}$ with 28 -ps pulses at a center wavelength of $\approx 960 \mathrm{~nm}$ [21]. In 2011, the first femtosecond VECSEL was demonstrated using QD-VECSEL modelocked by a QD-SESAM [22]. Already in 2012, ultrafast VECSELs generated 5.1 W with 682 -fs pulses [23]. In the sub-500-fs regime, $150 \mathrm{~mW}$ was obtained [22]. Even shorter pulse durations down to $107 \mathrm{fs}$ with an average output power of $3 \mathrm{~mW}$ were demonstrated [24] in fundamental modelocking or down to $60 \mathrm{fs}$ in a multipulsing mode [25], showing the potential of this technology.

However, for stabilizing the frequency comb of a modelocked VECSEL, sub-300-fs pulses in combination with high-average output powers are important. A quantitative understanding of the pulse formation process is essential in order to identify and optimize the laser parameters, which currently limit high-power performance in the sub-300-fs regime. So far, most of the studies on the pulse formation of ultrafast VECSELs focused on the picosecond regime. In 2010, we verified our model of a soliton-like pulse shaping mechanism in this regime by comparing simulations to measurement results [26, 27]. Our numerical model describes the interplay of nonlinear phase shifts, induced by strong gain and absorber saturation, with positive intra-cavity group delay dispersion (GDD). In our simulation model, a pulse is represented by its complex envelope, and the pulse is iterated inside the cavity. Its strength is the direct connection to macroscopic measurable parameters such as modulation depth and saturation fluence. Previously, quantitative predictions of pulse formation and resulting pulse durations in the femtosecond region were not possible, because the most important experimental parameters (including the bandwidth and saturation fluence of the gain) were unknown. In very recent work, these parameters were accurately characterized [28]. By combining this information with the well-known parameters of the cavity and the saturable absorber [29, 30], it is now possible to investigate the pulse formation mechanism in the femtosecond regime.

In this paper, we analyze in detail, both numerically and experimentally, femtosecond pulse formation in VECSELs. We identify the limiting parameters for pulse duration and pulse energy and develop guidelines for future high-power VECSELs operating with pulse durations of a few 100-fs. We show that GDD management is extremely important to achieve short pulses of a few 100-fs. Because of this strong influence of the GDD, we designed a quantum well SESAM (QW-SESAM) with fast recovery dynamics and a low-dispersion top coating similar to the ones we used for recent VECSEL designs [22, 31]. In combination with the same QD-VECSEL as used in [22] for the generation of 784-fs pulses, we were able to realize an intra-cavity dispersion of about $50 \mathrm{fs}^{2}$ which led to 364-fs pulses at an average output power of $70 \mathrm{~mW}$. This result was obtained with a cavity geometry using the same mode size on the gain and the absorber, usually referred as " $1: 1$ modelocking" [2]. This configuration is important because it paves the way toward an integrated MIXSEL design. We simulate this result with our model, showing an excellent quantitative agreement for the pulse duration, output power as well as spectral bandwidth. Furthermore, we verify our model by analyzing numerically a recently published femtosecond VECSEL, for which the repetition rate was tuned over a range from 6.5 to $11.3 \mathrm{GHz}$ by just changing the cavity length [31]. Over the full range of energy changes (of about a factor of 2), we obtain an excellent agreement between theory and experiment.

With the experimental verification of our numerical model, we therefore have a simple but powerful tool to investigate further pulse shortening and power scaling techniques. To evaluate guidelines for future optimized VECSEL and SESAM designs, we investigate the impact of different parameters of the gain and the SESAM on the pulse duration as well as the output power. We show that especially the saturation fluence of the gain is an important key parameter to obtain shorter pulses combined with a high-average output power. Taking all those guidelines into account, our model predicts 200 -fs pulses with an average output power of more than $1 \mathrm{~W}$, which should be feasible to generate a coherent supercontinuum and to stabilize the frequency comb.

\section{Numerical modeling of the pulse formation in SESAM-modelocked VECSELs}

In this section, we develop the model, in particular showing how the evolution of the electric field in the laser cavity can be represented in terms of macroscopic and measurable parameters. This allows us to directly investigate the influence of these parameters on the pulse buildup with only a few assumptions, since most of the parameters are well known and can be easily measured [28-30].

\subsection{Numerical representation of the pulse}

In our model, we use the slowly varying envelope approximation (SVEA) to represent the pulse of the electric field in the time domain: 


$$
\begin{aligned}
& E(t)=\operatorname{Re}\left\{A(t) e^{-i \omega_{0} t}\right\} \\
& P(t)=|A(t)|^{2},
\end{aligned}
$$

where $E(t)$ represents the temporal electric field adapted to have units $\sqrt{W}, P(t)$ the instantaneous power and $\omega_{0}$ the reference frequency, usually chosen to be close to the center frequency of the pulse. The pulse is represented within a temporal window $T$ from $[-T / 2, T / 2)$, with the temporal resolution, denoted $\delta t$, given by the number of sampling points. The pulse is then propagated through the laser cavity by iterating over all of the intra-cavity elements, including the gain, saturable absorber, output coupler, etc. The effects of the different intra-cavity elements are applied on the pulse envelope by numerical operators, either in time or in frequency domain, whichever is more suitable. With fast Fourier transform (FFT) algorithm, the pulse envelope can be easily transformed between the time and the frequency domain. The FFT implies a spectral resolution of $T^{-1}$. As a guideline, the time resolution and the time window $T$ are chosen in a way that the main part of the pulse envelope is sampled with at least 20 sampling points in both time and frequency domain. For more details on the numerical implementation, we refer to $[26,32]$ where the principles underlying of our model were first introduced and described.

We implemented the model using the Python programming language and the numerical libraries NumPy and SciPy. The computation of the pulse formation in a given cavity usually takes less than a minute and can be done with a desktop computer. To investigate the influence of different parameters, as done in this paper, usually a huge number of single pulse formation simulations are needed. We use the pp (Parallel Python) package to run many simulations in parallel to decrease the calculation time.

A typical modelocked VECSEL cavity consists of an output coupler, a gain chip acting as a folding mirror, and a SESAM (see Fig. 1a). In order to model these intracavity elements, it is necessary to take several effects into account. These effects are shown schematically in Fig. $1 \mathrm{~b}$ and will be explained in detail in the following subsections.

\subsection{Gain}

The gain is implemented with three operators representing the effects of gain saturation, wavelength filtering, and noise (see Fig. 1b).

Dynamic gain saturation [33] of a pulse is an important effect. Due to the short lifetime and the high gain cross section of the semiconductor gain, the gain is saturated strongly during the pulse, but also recovers rapidly compared to bulk solid-state lasers materials. This process is described by the differential equation relying on the rate equations of a two-level system [26, 34]:

$$
\frac{\mathrm{dg}(\mathrm{t})}{\mathrm{d} t}=\underbrace{-\frac{g(t)-g_{\mathrm{ss}}}{\tau_{g}}}_{\text {recovery }} \underbrace{-g(t) \cdot \frac{P(t)}{E_{\mathrm{sat}, g}},}_{\text {saturation }}
$$

where $E_{\mathrm{sat}, g}$ is the saturation energy, $g(t)$ the power gain, $g_{\mathrm{ss}}$ the small-signal gain (respectively, the unsaturated gain), $\tau_{g}$ the recovery time of the gain, and $P(t)$ is the optical power of the pulse envelope inside the cavity. The first part of this differential equation describes the recovery of the gain, while the second part describes the gain saturation. Numerically, we determine the gain $g(t)$, by solving Eq. 2, using the specified time window $[-T / 2, T / 2]$, and the power $P(t)$ of the pulse incident on the gain element.

The saturation of the semiconductor gain material also implies a change of the real part of the refractive index due to the Kramers-Kronig relation. Therefore, an additional phase change is applied given by

$\Delta \varphi(t)=-\frac{\alpha_{g}}{2}(t)$,

where $\alpha_{g}$ is the phenomenological linewidth enhancement factor (LEF) for the gain [35].

The spectral gain filter is implemented by a parabolic filter function, representing the curvature of the gain profile, and acts on the envelope of the pulse in the frequency domain. We use this approach because the curvature of the gain profile, and not the actual gain bandwidth, is the physical quantity responsible for filtering and stretching the pulse [36]. A parabolic gain filter corresponds to a squared parabola for gain profile measurements, which is an
Fig. 1 a Typical modelocked VECSEL cavity showing the cavity elements and $\mathbf{b}$ its implementation in our numerical model: A pulse circulates in the cavity with all the effects of the cavity elements taken into account
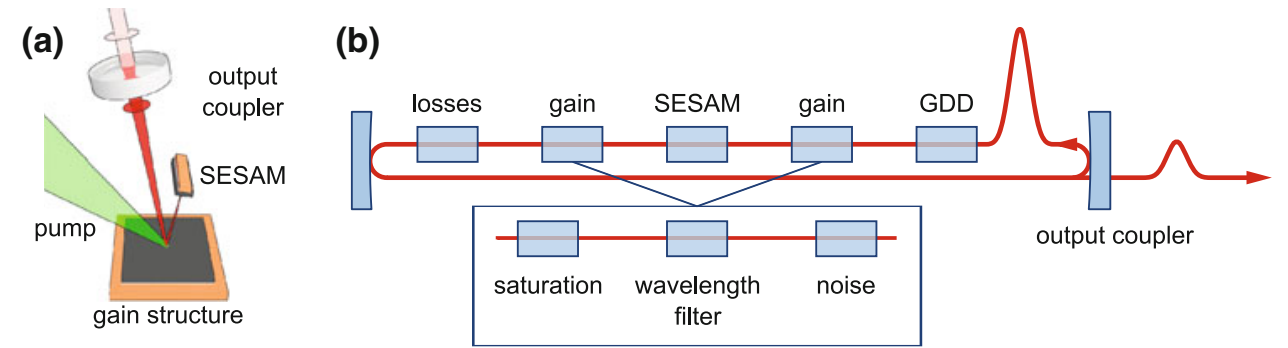
excellent agreement with our experimental data [28] (see Fig. 2a). Since the amplification is already implemented in the saturation operator described above, this squared parabola is normalized (blue dashed). The final parabolic filter function is the square root of this normalized squared parabola since it is applied to the amplitude pulse envelope. Note that the effective FWHM of the filter function is much bigger than the FWHM of the gain profile. For example, a FWHM of $10 \mathrm{~nm}$ for the gain profile corresponds to about $50 \mathrm{~nm}$ FWHM of the parabolic filter function. These values are comparable to the ones measured in [36], where they also used a parabolic approach for the filter function. For our structures, we measured 25-30 nm FWHM for the gain profile, which corresponds to larger values of the FWHM of the parabolic filter of more than $100 \mathrm{~nm}$ (see Fig. 2b).

With the gain, also spontaneous emission is taken into account as noise by adding random complex amplitudes with a variance of $\sigma^{2}=P_{\text {noise }} / 2$. We usually use noise floor powers up to $10 \mathrm{nW}$.
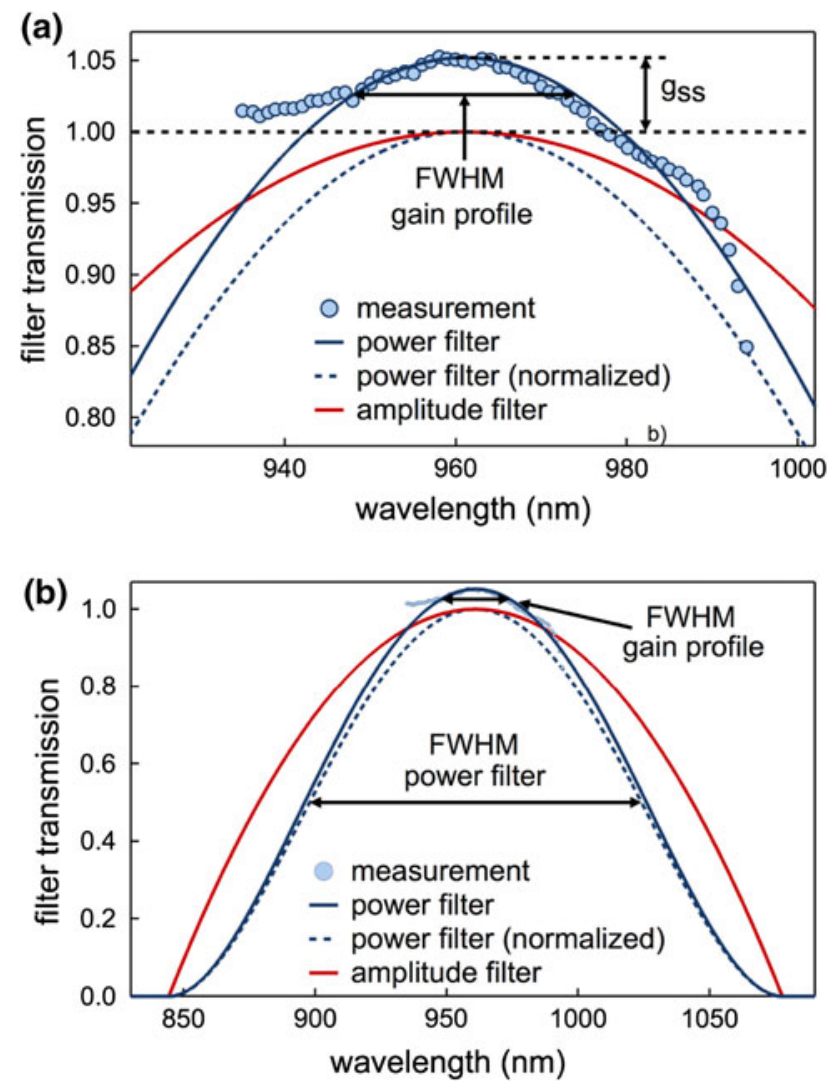

Fig. 2 a Parabolic amplitude filter (red) as used in the simulations. This filter is derived from the squared parabola, matching the spectral gain measurements, which is then normalized. b The effective FWHM of the filter function is much bigger than the FWHM of the gain profile

\subsection{SESAM}

The saturation effects of the saturable absorber, in our case a SESAM, are implemented with the same operator as the gain saturation. As a simple approximation, the small-signal gain gss, introduced in Eq. 2, can be replaced with $-\Delta R$, where $\Delta R$ is the modulation depth of the SESAM. This results in the following differential equation similar to Eq. 2:

$\frac{\mathrm{dq}(\mathrm{t})}{\mathrm{d} t}=\underbrace{-\frac{q(t)+\Delta R}{\tau_{a}}}_{\text {recovery }} \underbrace{-q(t) \cdot \frac{P(t)}{E_{\mathrm{sat}, a}},}_{\text {saturation }}$

where $E_{\text {sat, } a}$ is the saturation energy of the absorber, $\tau_{a}$ the recovery time, and $q(t)$ the power loss of the absorber. As shown in Fig. 3, pump-probe measurements of the recovery dynamics of a SESAM show two clearly distinguishable recovery processes, which can be fitted well with a double exponential fit with two time constants [37]:

$\Delta R_{\mathrm{PP}}(t)=A \cdot e^{-t / \tau}+(1-A) \cdot e^{-t / \tau_{\text {fast }}}$

where $A$ is the amplitude of the component with slow time constant $\tau_{\text {slow }}$ and $(1-A)$ the amplitude of the component with the fast time constant $\tau_{\text {fast }}$. The measurement of a SESAM is shown in Fig. 3 (blue dots) with an amplitude $A$ of $73 \%, \tau_{\text {slow }}$ of $71.8 \mathrm{ps}$ and $\tau_{\text {fast }}$ of $1.1 \mathrm{ps}$. Using a simplified formula with only a single time constant, according to the recovery part of Eq. 4, does not describe the system accurately enough. This is illustrated with the simulations of a pump-probe measurement shown in Fig. 3 (solid and dashed blue lines).

Similar to the differential equation for the gain, the recovery and the saturation part of Eq. 4 are consecutively solved and applied for each step $\delta t$ to the pulse envelope in

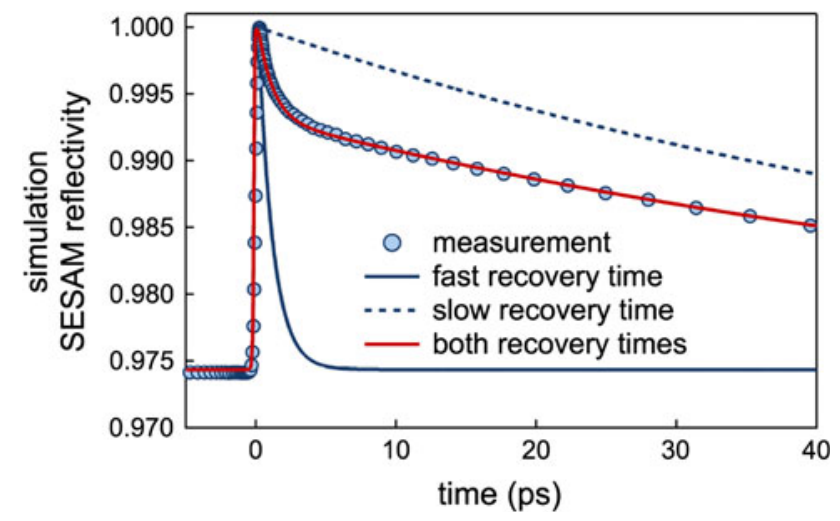

Fig. 3 Recombination of the SESAM is implemented in the model with two recombination times, which is in good agreement with pump-probe measurements. In comparison with only the fast recombination or the slow recombination time, which are not appropriate enough 
the time domain. Solving the saturation part first makes it possible to separate the recovery part of Eq. 4 and modify it to match the double exponential behavior (red line). We implemented this recovery behavior by splitting $q(t)$ into two parts $q_{\text {slow }}(t)$ and $q_{\text {fast }}(t)$ with

$$
\begin{aligned}
q_{\text {slow }}(t) & =A \cdot q(t) \\
q_{\text {fast }}(t) & =(1-A) \cdot q(t) .
\end{aligned}
$$

This approach does not influence the saturation part of Eq. 4, which is solved first. However, the recovery part of Eq. 4 can be replaced by two different equations for $q_{\text {slow }}(t)$ and $q_{\text {fast }}(t)$ :

$$
\begin{aligned}
\frac{\mathrm{dq}_{\text {slow }}(\mathrm{t})}{\mathrm{d} t} & =-\frac{q_{\text {slow }}(t)+A \cdot \Delta R}{\tau_{\text {slow }}} \\
\frac{\mathrm{dq}_{\text {fast }}(\mathrm{t})}{\mathrm{d} t} & =-\frac{q_{\text {fast }}(t)+(1-A) \cdot \Delta R}{\tau_{\text {fast }}} .
\end{aligned}
$$

Furthermore in a SESAM, the saturation implies a change on the real part of the refractive index due to the Kramers-Kronig relation. Therefore, an additional phase change given by Eq. 3 is applied with the corresponding LEF $\alpha_{a}$ for the SESAM.

\subsection{Group delay dispersion}

Group delay dispersion (GDD) is important, since it can have a huge influence on the pulse duration. In our model, a wavelength-independent GDD value is applied to the pulse envelope in the frequency domain. Its phase shift is given by

$\Delta \phi(\omega)=\frac{1}{2} \cdot D \cdot\left(\omega-\omega_{0}\right)^{2}$,

where $D$ is the dispersion coefficient, usually expressed in $\mathrm{fs}^{2}$ and $\omega_{0}$ the reference frequency.

\subsection{Additional operators}

Internal losses, such as scattering, spontaneous emission as well as losses due to the output coupler and nonsaturable losses of the absorber are accounted for either in the time domain or in the frequency domain by simply adding a constant loss to the envelope.

The SESAM absorbs the leading edge of the pulse, which results in a shift backward in time. This effect is stronger than the shift forward given by the gain saturation, where the leading edge of the pulse is amplified more than the trailing edge. To avoid the pulse running toward the end of its grid, a centering operator is applied every 10 roundtrips to center the pulse to $t=0$.

\subsection{Input parameters}

Our model is strongly based on macroscopic, measurable input parameters. All used parameters are listed in Table 1. The recovery dynamics as well as the saturation behavior of the SESAM are well known and can be measured in our laboratory $[29,30]$. Cavity parameters are usually well known from the resonator design. We measured the GDD of our samples by white light interferometry [38, 39]; however, measurements are limited by a measurement error of about $\pm 100 \mathrm{fs}^{2}$. We therefore calculated the GDD from the designs of the included low-dispersion multilayer semiconductor structures. With the recent characterization of our gain structures [28], we now know the saturation fluence, the gain curvature (see Table 1), and the small-signal gain. These measurements have been done with pump intensities, which are similar to the pump intensities used in the femtosecond laser we model in Sect. 4. The gain recovery time is assumed to be in the nanosecond time range [40].

If not explicitly mentioned differently, all further simulations in this paper are done with the parameters listed in Table 1.

\subsection{Simulations}

The simulations are usually started from a $10 \mathrm{nW}$ noise floor and are stopped if a stable solution is found as

Table 1 Input parameters used for the simulation and the corresponding simulation result

\begin{tabular}{llllll}
\hline Parameter & Gain & Abs. & Cavity parameters & \multicolumn{2}{l}{ Results } \\
\hline Saturation fluence $\left(\mu \mathrm{J} / \mathrm{cm}^{2}\right)$ & 45 & 5 & Repetition rate $(\mathrm{GHz})$ & 3.97 & Av. output power $(\mathrm{mW})$ \\
Relaxation time $\tau_{g}(\mathrm{~ns})$ & 3 & - & Other losses $(\%)$ & 0.8 & FWHM pulse dur. (fs) \\
Fast recovery time $\tau_{\text {fast }}(\mathrm{fs})$ & - & 430 & Output coupler $(\%)$ & 1.0 & FWHM spectrum $(\mathrm{nm})$ \\
Slow recovery time $\tau_{\text {slow }}(\mathrm{ps})$ & - & 9 & Center wavelength $(\mathrm{nm})$ & 966 & TBP $\left(\times \operatorname{sech}^{2}\right)$ \\
Amplitude of slow comp. $(\%)$ & - & 50 & Cavity GDD $\left(\mathrm{fs}^{2}\right)$ & 50 & \\
Modulation depth $\Delta R(\%)$ & - & 1.8 & Noise floor power $(\mathrm{nW})$ & 10 & \\
Beam radius $r(\mu \mathrm{m})$ & 120 & 120 & & & \\
Linewidth enhancement factor $\alpha$ & 3 & 2 & & & \\
Small-signal gain $g_{\text {ss }}$ & 5 & - & & & \\
FWHM gain bandwidth $(\mathrm{nm})$ & 26 & - & & & \\
\hline
\end{tabular}

In this case, measurements of a VECSEL with QD layers and a SESAM with one single QW absorber are listed 
(a)

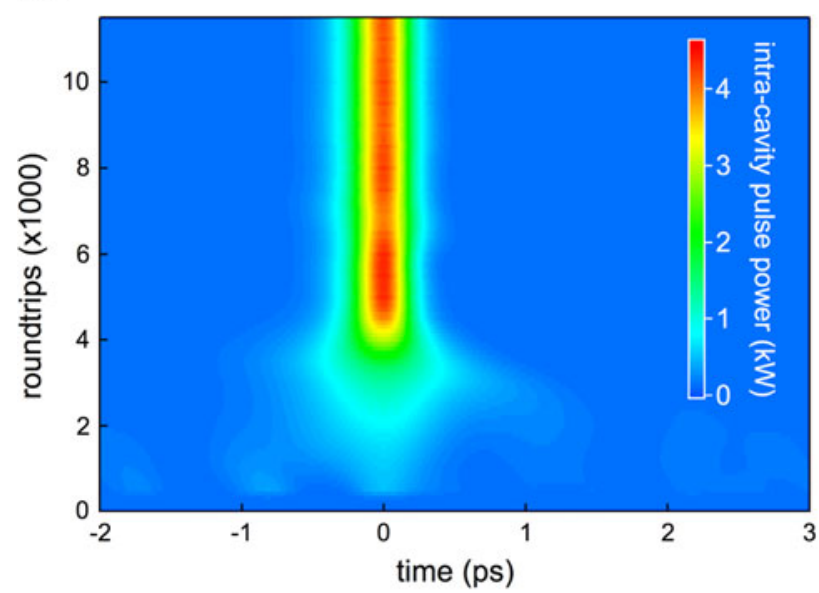

Fig. 4 a Pulse buildup from a $10 \mathrm{nW}$ noise floor showing the intracavity power distribution and its corresponding $3 \mathrm{D}$ representation. The pulse has a duration of $364 \mathrm{fs}$ and is stable after about 104 roundtrips. The simulation finished after $\mathbf{b}$ the pulse duration,

illustrated in Fig. 4a. We define a pulse as stable if the pulse duration, the spectral width, the peak power, and the pulse energy are all varied by $<0.5 \%$ within the previous 3,000 roundtrips. The pulse in Fig. 4 is stable after about $10^{4}$ roundtrips, which corresponds to about $3 \mu \mathrm{s}$ in case of a $3.97 \mathrm{GHz}$ laser. It is also possible to start the simulations with an initial $\operatorname{sech}^{2}$-pulse. This shortens the calculation time and delivers the same result as starting from noise. Figure $4 \mathrm{~b}-\mathrm{e}$ compares a simulation started from noise (red lines) to one started with an initial 1-ps soliton pulse (blue lines) regarding the pulse properties used for the stability criteria mentioned above. In comparison with the pulse initialized from a soliton, the noise-initialized pulse takes about $50 \%$ more roundtrips until it is stable. Thus also, the computational effort is higher.

\section{Modelocking mechanism in the femtosecond regime}

The combination of saturable gain and saturable absorber leads to a soliton-like modelocking mechanism which was first introduced for SESAM-modelocked VECSELs in [26]. The dynamic behavior of the gain and the absorber leads to a total phase shift, which is similar, compared to the one induced by SPM in soliton modelocked ion-doped solidstate lasers [41, 42], but with opposite sign. In soliton modelocking, the phase change induced by SPM can be balanced with negative GDD to obtain stable soliton pulses. In analogy, positive GDD is required to balance these phase shifts in ultrafast VECSELs to obtain stable modelocked operation with short pulses. This mechanism is (b)

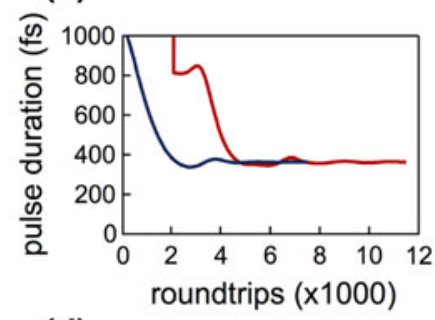

(d)

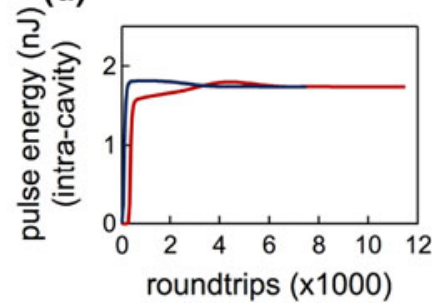

(e)

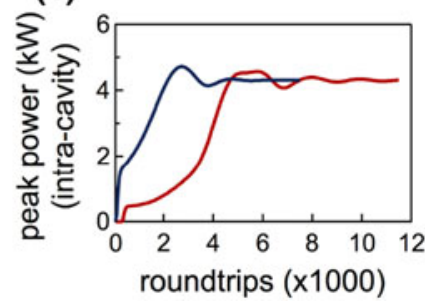

c spectral width, d the pulse energy, and e the peak power varied $<0.5 \%$ within the last 3,000 roundtrips (red lines). Starting from an initial 1-ps-pulse (blue lines) shortens the calculation time but delivers the same result as started from noise

called soliton-like modelocking and was experimentally verified in the picosecond regime by comparing simulations to real measurements [27].

The phase shifts described above are illustrated in Fig. 5a for a sub-500-fs pulse. These phase shifts are usually in the range of a few mrads. From the picosecond regime [27], it is known that these phase changes induce an asymmetric behavior of the pulse duration as a function of the intra-cavity GDD. To explore this, we simulated the influence of GDD on the pulse duration using the parameters given in Table 1. Additionally, we also varied the gain bandwidth ranging from $5 \mathrm{~nm}$ up to $40 \mathrm{~nm}$ with the results illustrated in Fig. 5b. For the picosecond experiment in [27], it was essential to keep the wavelength stable. This was done using an intra-cavity etalon. As mentioned before, the gain bandwidth is realized by a filter function. Therefore, an etalon can be approximated by a small gain bandwidth of, for example, $5 \mathrm{~nm}$, which is plotted in Fig. 5c (solid blue). It shows clearly that femtosecond operation is suppressed, and the influence of GDD is slightly asymmetric for the given GDD range. Compared to the study in [27], this influence is small, but the GDD range in this study was also much larger $\left( \pm 2 \times 10^{4} \mathrm{fs}^{2}\right)$. Nevertheless, in this small range of $\pm 1,000 \mathrm{fs}^{2}$, it is obvious that slightly positive dispersion leads to shorter pulses in the picosecond regime, while negative dispersion increases the pulse duration drastically. By increasing the gain bandwidth, femtosecond pulses can be obtained. For example, at a gain bandwidth of $25 \mathrm{~nm}$, which corresponds to the gain bandwidth of our structures [28], even sub-300fs pulses are obtained from our simulations. However, the 

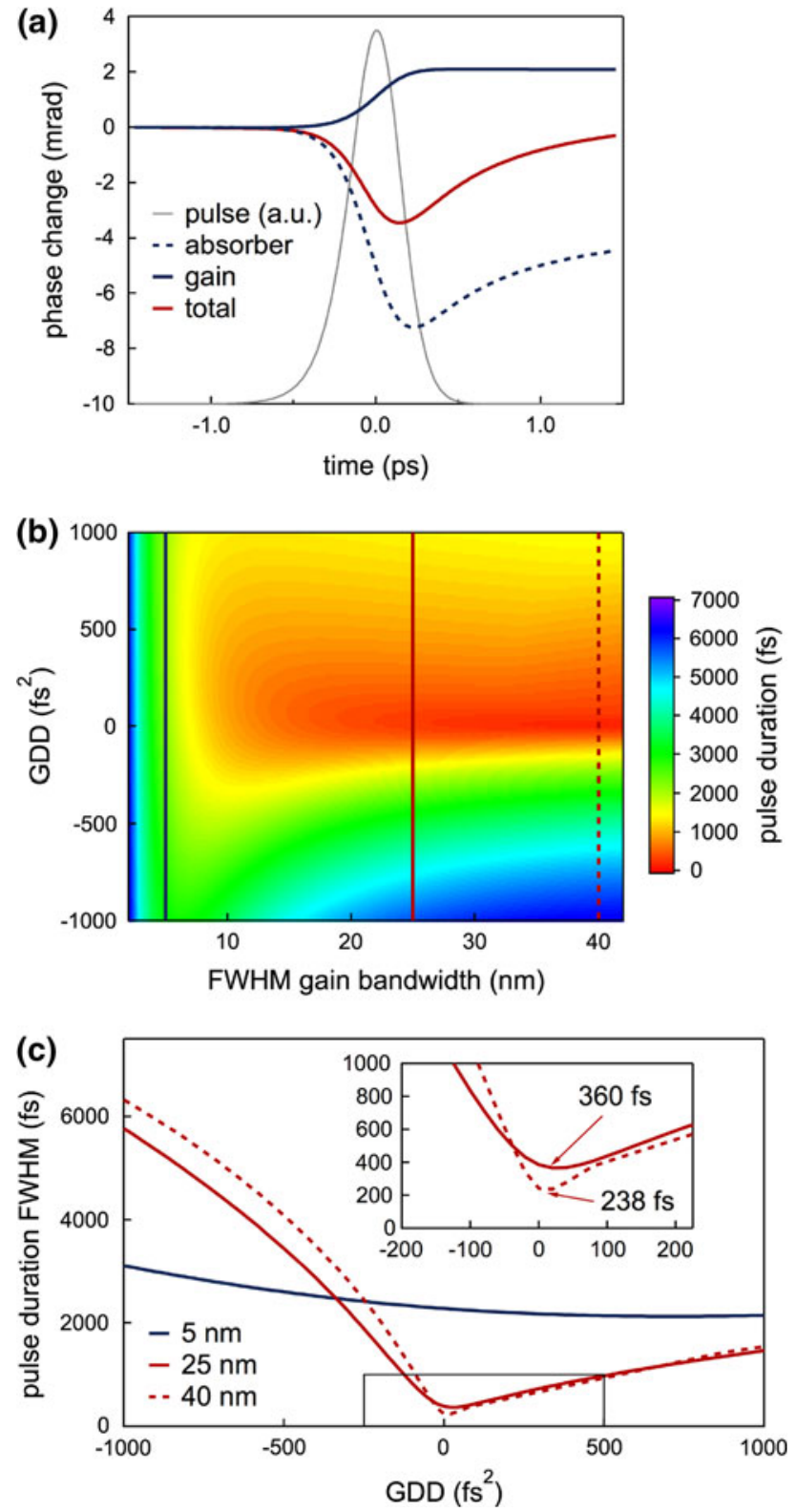

Fig. 5 a Time-dependent phase change of a simulated 364-fs pulse showing the overall phase change consisting of the phase changes induced by saturation of the gain and the absorber. This phase change is similar to SPM but with a negative sign. b Simulated pulse duration in dependence of the gain bandwidth and intra-cavity GDD showing an asymmetrical dependence on the GDD. $\mathbf{c}$ Cross profiles of $\mathbf{b}$ at a gain bandwidth of 5, 25, and $40 \mathrm{~nm}$ the influence of GDD on the pulse duration. For fs pulses, it is essential to operate at minimized positive GDD in the cavity

influence of GDD is very strong, and it is very important to have a small and positive dispersion in the cavity. Small negative dispersion values of $<-200 \mathrm{fs}^{2}$ already lead to picosecond pulses. With an increased bandwidth of $40 \mathrm{~nm}$, pulses as short as $238 \mathrm{fs}$ are predicted by the model. Our model predicts a strong influence of GDD on the pulse formation process and that precise dispersion control is even more important in the femtosecond regime.

\subsection{Linewidth enhancement factor}

The phase changes of an ultrafast VECSEL as illustrated in Fig. $5 \mathrm{a}$ are given by Eq. 3. We usually assume the LEF $\alpha_{g}=3$ for the gain and $\alpha_{a}=2$ for the absorber and that they are constant while the pulse passes through. It is difficult to reliably measure values under the same condition as in modelocked operation, since the LEF for the gain and the absorber are wavelength and carrier dependent. It has been reported that different measurement methods can also lead to different values, especially for QD layers [43]. Hence, due to this lack of exact values, it is worthwhile to investigate the influence of the different LEFs on the pulse duration. A simulation in Fig. 6 shows that these LEFs are quite uncritical for a broad range of values for $\alpha_{g}$ and $\alpha_{a}$, regarding the pulse duration (Fig. 6a) as well as the output power (Fig. 6b). In theory, changes of the LEF during the interaction of the pulse with the gain medium have been reported [44]. Simulations with a nonconstant LEF have been already done, still showing the soliton-like modelocking behavior [26]. This can be explained because the changes of the LEFs are similar for the gain and the absorber and since the resulting phase shifts are opposite to each other, the total phase shift is not influenced that much.

In spite of those uncertainties, we report on very good quantitative agreement of the simulations compared to recent experimental results in Sect. 4, even with the assumption of a constant LEF.

\section{Experimental verification}

In our model, almost all input parameters are experimentally measurable. While the parameters of the SESAM, like modulation depth, saturation fluence, fast and slow recovery time can be measured accurately for many years now, experimental parameters for the gain were not available until very recently [28]. Without knowing all important parameters in detail, the simulations only allowed us to investigate the principles of the pulse forming mechanism but no quantitative predictions in femtosecond operation. An important improvement of our simulation model was the accurate measurement of these gain parameters. In contrast to previously assumed saturation fluences of $160 \mu \mathrm{J} / \mathrm{cm}^{2}$ for the gain, we measured $30 \mu \mathrm{J} / \mathrm{cm}^{2}$ to 80 $\mu \mathrm{J} / \mathrm{cm}^{2}$ for our structures. The gain bandwidth and the gain saturation almost complete the gain parameters, which we implemented in our model as described above in Sect. 2.2.

The simulations of Fig. 5b show a strong influence of GDD on the pulse duration. We already realized both 

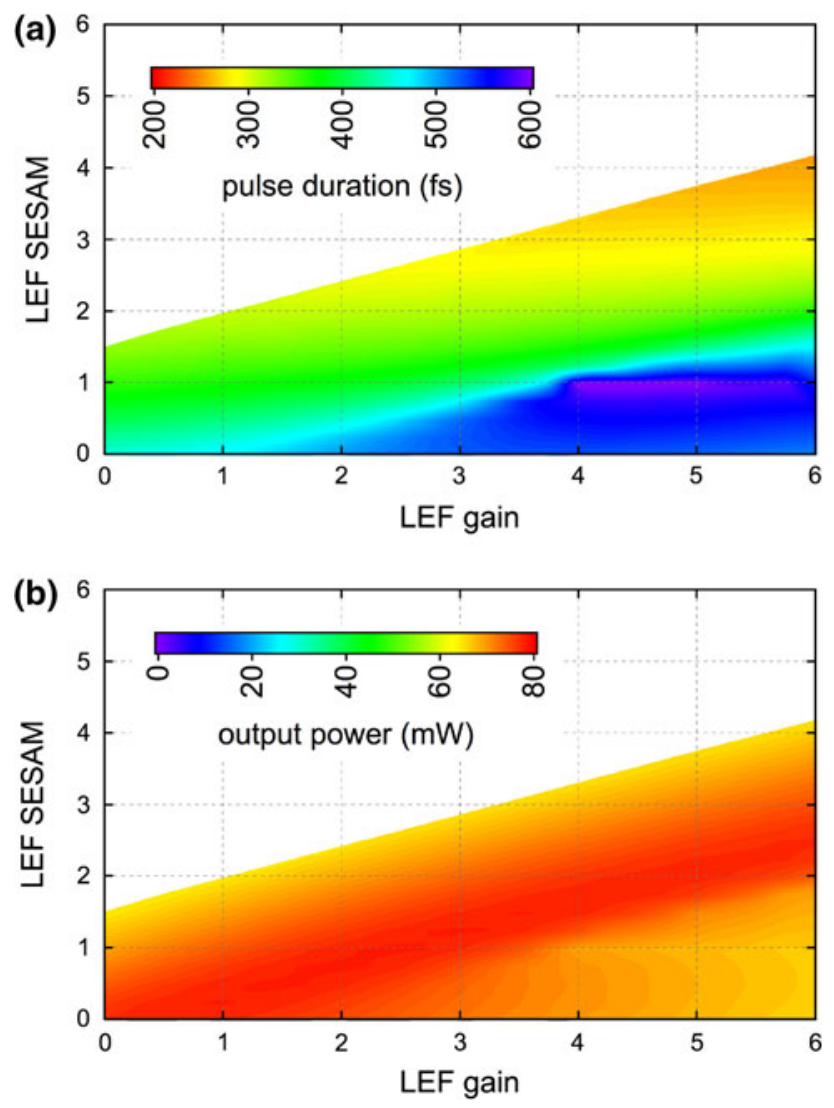

Fig. 6 Influence of the linewidth enhancement factor (LEF) on the pulse duration (a) and the output power (b). There is a band in which the influence of the LEF is uncritical for both pulse duration and output power. In the white area, the pulses were not stable

QW- and QD-VECSEL structures with a low-dispersion top coating leading to femtosecond operation [22, 31]. Up to that point, the dispersion of the SESAM was negligible compared to the one of the VECSEL gain structure. Because of this, we designed a new QW-SESAM using the same low-dispersion top coating as reported in [22]. The SESAM consists of one low-temperature grown InGaAs QW-layer embedded in AlAs, which resulted in a fast recovery time of $430 \mathrm{fs}$. With this new SESAM in combination with a QD-VECSEL (same as in [22]), we were able to obtain 364-fs pulses with an average output power of $70 \mathrm{~mW}$ and a repetition rate of $3.97 \mathrm{GHz}$ (see Fig. 7). The cavity was a simple v-shaped cavity (Fig. 1a, consisting of the QW-SESAM, the QD-VECSEL, and output coupler. Using a radius of curvature of $200 \mathrm{~mm}$ for the output coupler results in a cavity configuration for which the laser mode radius on the gain structure and the SESAM was $120 \mu \mathrm{m}$. The corresponding SESAM and gain characterization measurements as well as the cavity parameters are summarized in Table 1. We calculated the intra-cavity dispersion to be slightly positive of about $50 \mathrm{fs}^{2}$. To our knowledge, this is the shortest pulse duration ever reached (a)

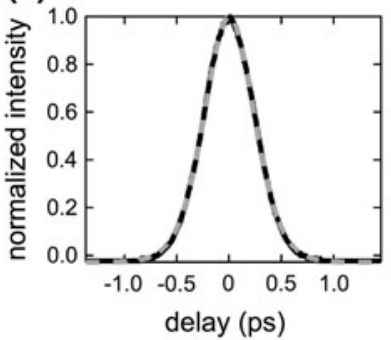

(b)
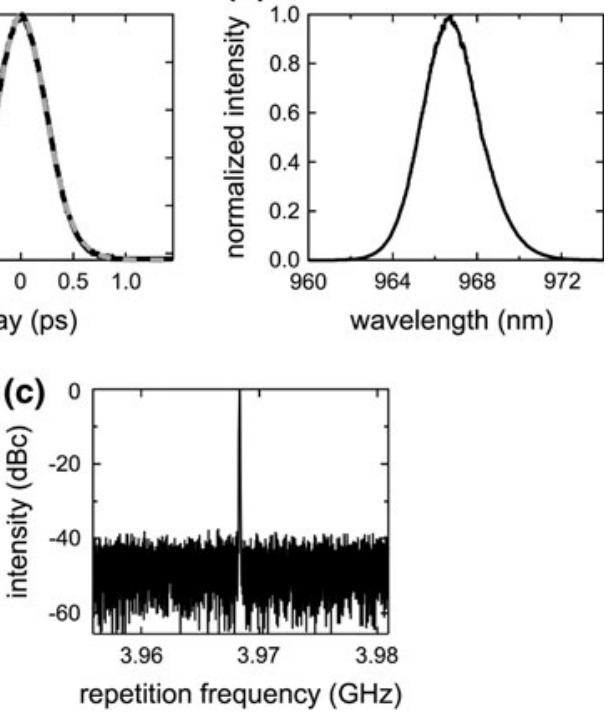

Fig. 7 Results obtained with a QD-VECSEL modelocked by a fast QW-VECSEL, both with a low-dispersion top coating. a Measured intensity autocorrelation trace (blue) and fitted autocorrelation of a 364-fs sech $^{2}$-pulse (dashed gray). b Measured optical spectrum with a spectral width of $3.04 \mathrm{~nm}$. c Microwave spectrum with a resolution bandwidth of $30 \mathrm{kHz}$ and a span of $25 \mathrm{MHz}$ showing a repetition rate of $3.97 \mathrm{GHz}$

Table 2 Experimental results compared to simulations

\begin{tabular}{lll}
\hline & Experiment & Simulation \\
\hline Av. output power $(\mathrm{mW})$ & 70 & 69 \\
FWHM pulse dur. (fs) & 364 & 363 \\
FWHM spectrum (nm) & 3.04 & 3.06 \\
TBP $\left(\times \operatorname{sech}^{2}\right)$ & 1.13 & 1.15 \\
\hline
\end{tabular}

in 1:1 modelocking and the shortest pulse duration using a QD-VECSEL.

Using exactly the same values given in Table 1, we were able to reproduce this result with our model numerically. Table 2 shows a comparison between experimental results and numerical simulations. There is impressive quantitative agreement for all of the key pulse parameters (pulse duration, output power, and spectral bandwidth).

Furthermore, we are now able to reproduce a recently published femtosecond QW-VECSEL result, where the repetition rate was tuned over a range from 6.5 to $11.3 \mathrm{GHz}$ [31]. In this large range of repetition rates, the pulse duration, as well as the average output power, remained nearly constant (Fig. 8 markers). This corresponds to significant pulse energy changes of about a factor of 2. The parameters given in [31] are summarized in Table 3 and were used to reproduce this result numerically (Fig. 8 solid lines). The wavelength in the experiment 
Table 3 Parameters used for the simulations to reproduce the experiment with the tunable repetition rates with a QWVECSEL and a QD-SESAM

\begin{tabular}{lllll}
\hline Parameter & Gain & Abs. & Cavity parameters & \\
\hline Saturation fluence $\left(\mu \mathrm{J} / \mathrm{cm}^{2}\right)$ & 35 & 3.8 & Repetition rate $(\mathrm{GHz})$ & $6.5-11.3$ \\
Relaxation time $\tau_{g}(\mathrm{~ns})$ & 10 & & Other losses $(\%)$ & 0.1 \\
Fast recovery time $\tau_{\text {fast }}(\mathrm{fs})$ & & 420 & Output coupler $(\%)$ & 2.0 \\
Slow recovery time $\tau_{\text {slow }}(\mathrm{ps})$ & & 15.6 & Center wavelength $(\mathrm{nm})$ & $963.6-964.1$ \\
Amplitude of slow comp. $(\%)$ & & 49 & Cavity GDD $\left(\mathrm{fs}{ }^{2}\right)$ & $300-200$ \\
Modulation depth $\Delta R(\%)$ & & 2.15 & Noise floor power $(\mathrm{nW})$ & 10 \\
Beam radius $r(\mu \mathrm{m})$ & 110 & 110 & & \\
Linewidth enhancement factor $\alpha$ & 3 & 2 & & \\
Small-signal gain $g_{\text {ss }}$ & 4.8 & & & \\
FWHM gain bandwidth $(\mathrm{nm})$ & 25 & & & \\
\hline
\end{tabular}

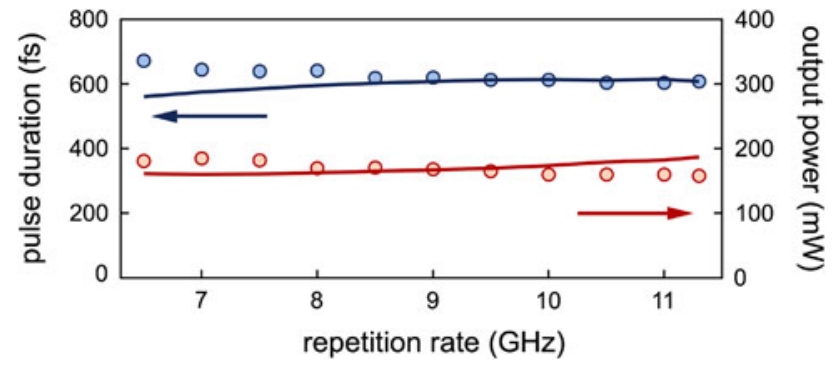

Fig. 8 Simulation of the pulse duration (blue) and average output power $(r e d)$ for various repetition rates compared to recently published measurements (markers). The model predicts the measurements extremely well over this wide range of repetition rates, corresponding to significant pulse energy changes of about a factor of 2

shifted slightly from $963.8 \mathrm{~nm}$ at $6.5 \mathrm{GHz}$ to $964.1 \mathrm{~nm}$ at $11.3 \mathrm{GHz}$. The GDD from the VECSEL structure is constant in this spectral range, due to the low-dispersion top coating. Based on calculations on the wavelength-dependent GDD from the design of the used SESAM structure, we implemented a $100 \mathrm{fs}^{2}$ GDD shift from 300 to $200 \mathrm{fs}^{2}$ within the mentioned spectral range. We obtained an excellent agreement with our experimental results for the pulse duration and the observed output power, as can be seen in Fig. 8.

The successful numerical reproduction of the 364-fs result as well as the experiment with the tunable repetition rates regarding the pulse duration and the observed output power is a quantitative verification of our numerical model in the femtosecond regime, which also confirms the soliton-like pulse shaping mechanism.

\section{Toward sub-300-fs high-power VECSELs}

Since our numerical model was experimentally confirmed in the femtosecond regime, we have a simple but powerful tool to investigate further pulse shortening and power scaling techniques. Thus, we investigated the impact of different parameters of the gain chip and the SESAM. Base parameters for all the following simulations are the ones we used to reproduce the 364 -fs result given in Table 1 . There are of course many different experimental scenarios, especially regarding the cavity geometry; however, we focused on the macroscopic parameters of the VECSEL and the SESAM. In all the simulations, we assumed 1:1 modelocking (same beam waist on VECSEL and SESAM) which was the case in our latest result and also because it would also be suitable for a femtosecond MIXSEL design. The goal is to provide a guideline for new VECSEL and SESAM designs to achieve high-power few-100-fs operation.

\subsection{Gain parameters}

We measured saturation fluences in the range of $30-80 \mu \mathrm{J} / \mathrm{cm}^{2}$ for our gain structures. These are lower values than we expected, which were in the order of $160 \mu \mathrm{J}$. While previous simulations mainly focused on the minimal achievable pulse duration in the picosecond regime, these new values even enabled us to predict the output power. It is therefore worthwhile to investigate the influence of these gain parameters, namely small-signal gain and saturation fluence. Figure 9a shows a simulation of the dependence of the pulse duration on these two gain parameters. The same simulation is shown in Fig. $9 \mathrm{~b}$ for the average output power. The effect of higher saturation fluence is illustrated in Fig. 9c where the pulse duration (blue) and the output power (red) are shown for constant small-signal gain values of $5 \%$ (solid) and $85 \%$ (dashed), corresponding to the horizontal colored lines in Fig. 9a, b. Please note that reaching the same small-signal gain for higher saturation fluence requires larger pump intensities. It is evident that the output power increases linearly with the saturation fluence, whereas the pulse duration decreases. Of course a higher small-signal gain at a given beam size and saturation fluence also increases the output power. 

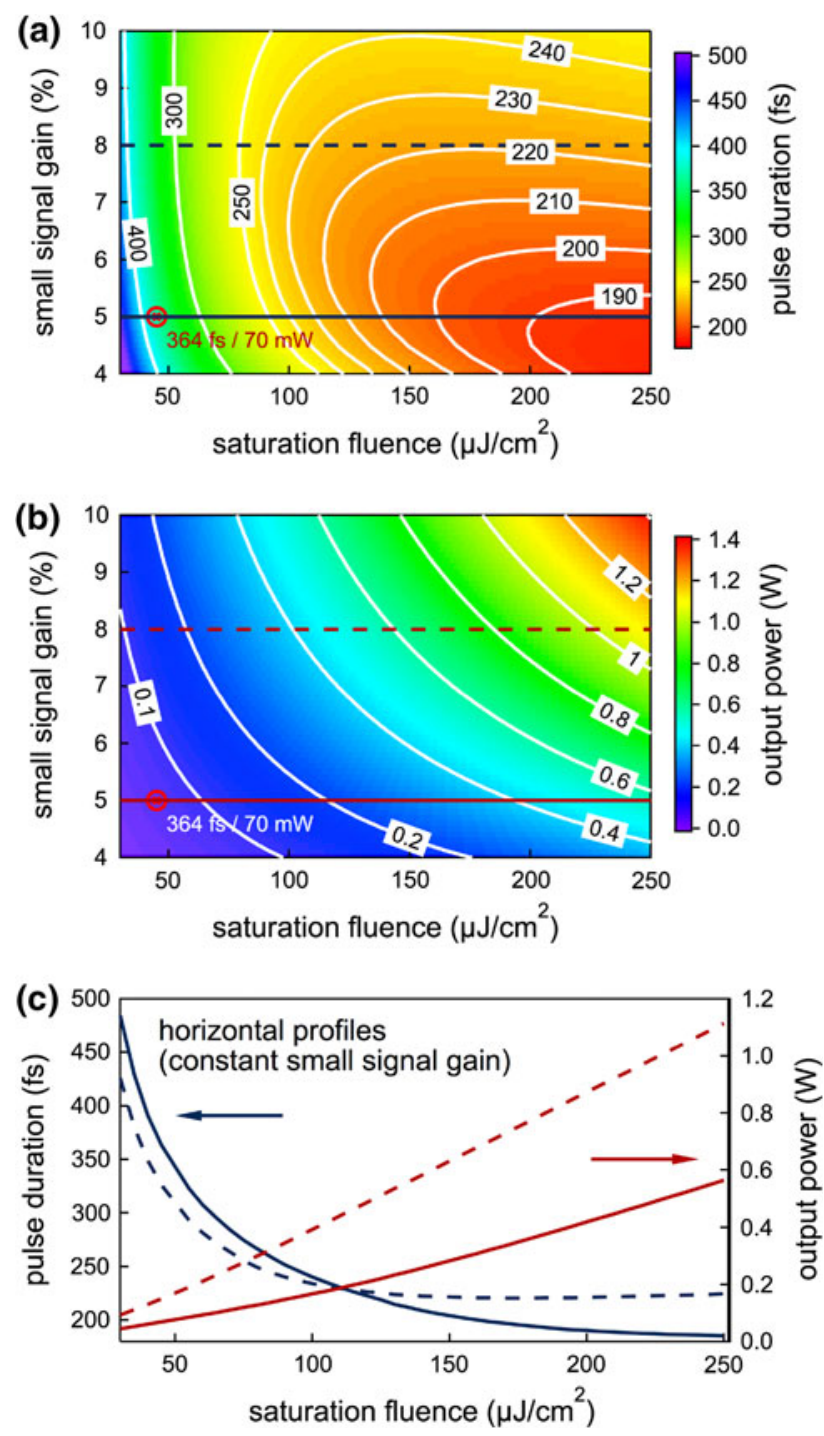

Fig. 9 Simulation on the influence of the saturation fluence and the small-signal gain on $\mathbf{a}$ the pulse duration and $\mathbf{b}$ the average output power. c Pulse duration (blue) and output power (red) for a fixed small-signal gain of $5 \%$ (solid) and $8 \%$ (dashed). The red points on both graphs show the corresponding parameters for the 364 -fs result

As a conclusion of these simulations, it is important for new designs to exhibit a higher saturation fluence to obtain a higher output power and shorter pulses. This can be done, for example, by placing the active QWs not in the antinodes of the standing wave pattern of the electric field, but somewhere in between or by lowering the field enhancement in the gain structure. This approach decreases the small-signal gain, but can be compensated by increasing the number of QWs. In this way, a saturation fluence above $100 \mu \mathrm{J} / \mathrm{cm}^{2}$ combined with $5-8 \%$ small-signal gain should be feasible. The influence of the gain bandwidth was already discussed in Sect. 3, and in Fig. 5b, it can bee seen that further increasing does not lower the pulse duration substantially.

\subsection{SESAM parameters}

In most of our experimental results, we used absorbers with very small saturation fluences $\left(\approx 5 \mu \mathrm{J} / \mathrm{cm}^{2}\right)$. One would expect that increasing the saturation fluence will lead to higher output power while the pulse duration remains stable. Therefore, we investigated the influence of the saturation fluence and the modulation depth of the SESAM on the pulse duration and the output power, which is shown in Fig. 10. Since no rollover [45] is implemented in the model, we took care, that in all cases, the saturation parameter did not exceed 20 [46]. This clearly shows that the shortest pulses can be realized by a SESAM with low saturation fluence (below $5 \mu \mathrm{J} / \mathrm{cm}^{2}$ ) and a high modulation depth. Regarding the output power, it is somehow a tradeoff, since a higher modulation depth causes lower output powers (Fig. 10b).

In the investigations of the recovery dynamics, we observed that the pulse duration is only minimally affected by the fast recovery time of the SESAM as it is illustrated in Fig. 11. This is somehow similar to the soliton modelocking mechanism where the recovery time of the SESAM also plays a minor role. Furthermore, we found that in our model, the slow recombination time does not affect the
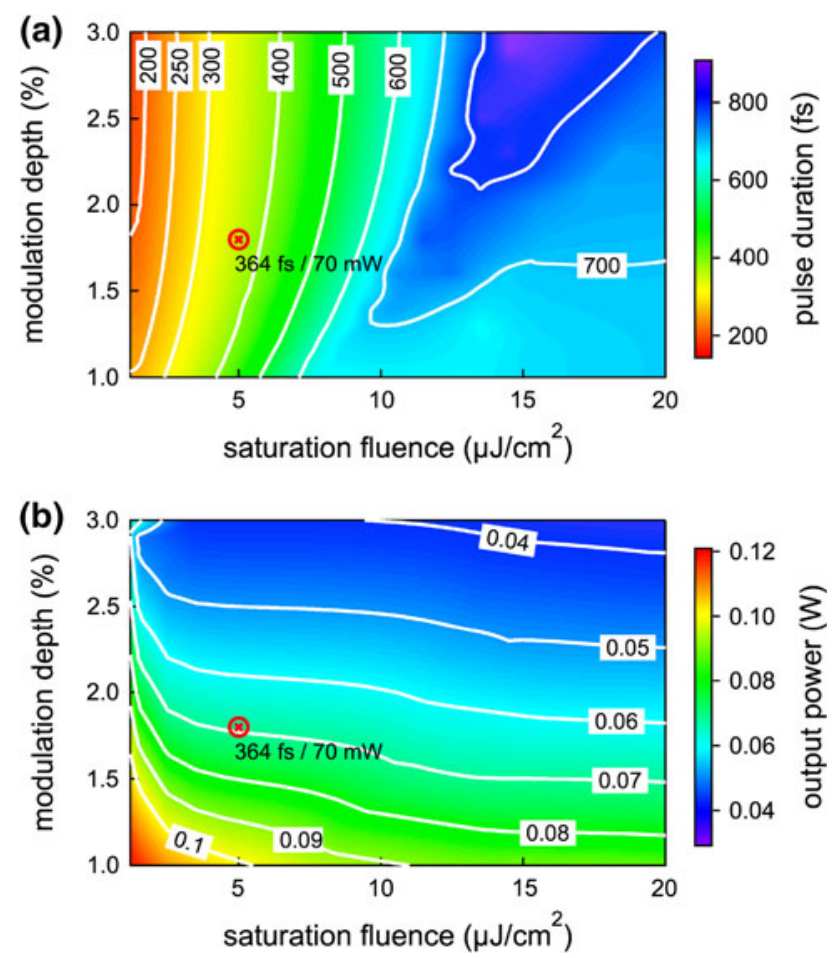

Fig. 10 Influence of the saturation fluence and the modulation depth of the SESAM on a the pulse duration and $\mathbf{b}$ the average output power. The shortest pulse can be realized with a low saturation fluence and a high modulation depth. It is a trade-off, since a high modulation depth decreases the output power. The red points on both graphs show the corresponding parameters for the 364-fs result 


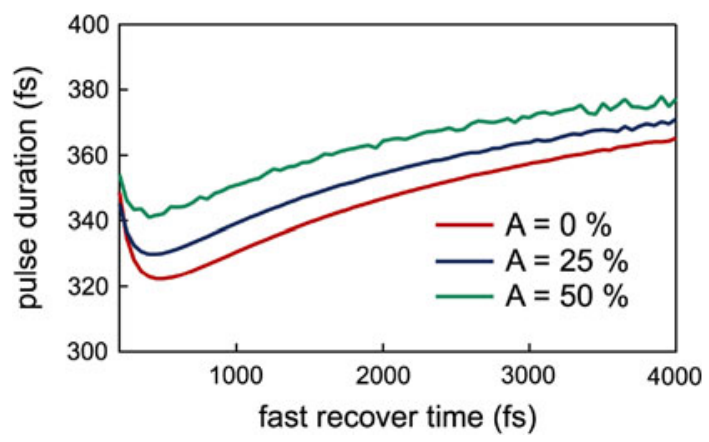

Fig. 11 Influence of the fast recovery time and the amplitude of the slow recovery time constant A on the pulse duration. The pulse duration is only slightly affected, but a higher amplitude destabilizes the pulse formation (see ripples)

pulse duration at all as long as the amplitude A of the slow recovery time constant, as introduced in Eq. 5, is below $50-60 \%$. If the amplitude is higher, the pulse formation is usually found to be unstable.

To summarize, for new SESAM design, it is important to have a low saturation fluence combined with a high modulation depth. In theory, the recovery dynamics seem to play a minor role. However, for all of our femtosecond results, it turned out that a fast SESAM is still necessary, which is contradictory to the simulations. All of these SESAMs had a fast recovery time constant below 1 ps. But the amplitude of the slow recovery time constant was always $<50 \%$. This is in good agreement with our simulations, which predict instabilities in the pulse forming for an amplitude of more than 50-60\%.

\subsection{Cavity parameters}

For modelocking, it is important to have a net gain window, which means that the SESAM has to saturate faster than the VECSEL. Figure 12 shows the simulation of the interplay between the saturation fluence of the gain and the absorber regarding the pulse duration and the output power. Higher saturation fluences of the gain relax the requirements on a low saturation fluence of the SESAM (Fig. 12a) as discussed above. The output power, however, mainly depends on the saturation fluence of the gain at a given beam area and small-signal gain (Fig. 12b).

\subsection{High-power femtosecond VECSEL}

Taking all the design guidelines presented above into account, we can design structures with the following parameters listed in Table 4. Since the simulations do not take thermal effects and rollover [45] into account, simple power scaling by increasing the spot sizes leads to a quadratic increase in the output power. But this is also necessary to obtain power levels in the watt regime. In this
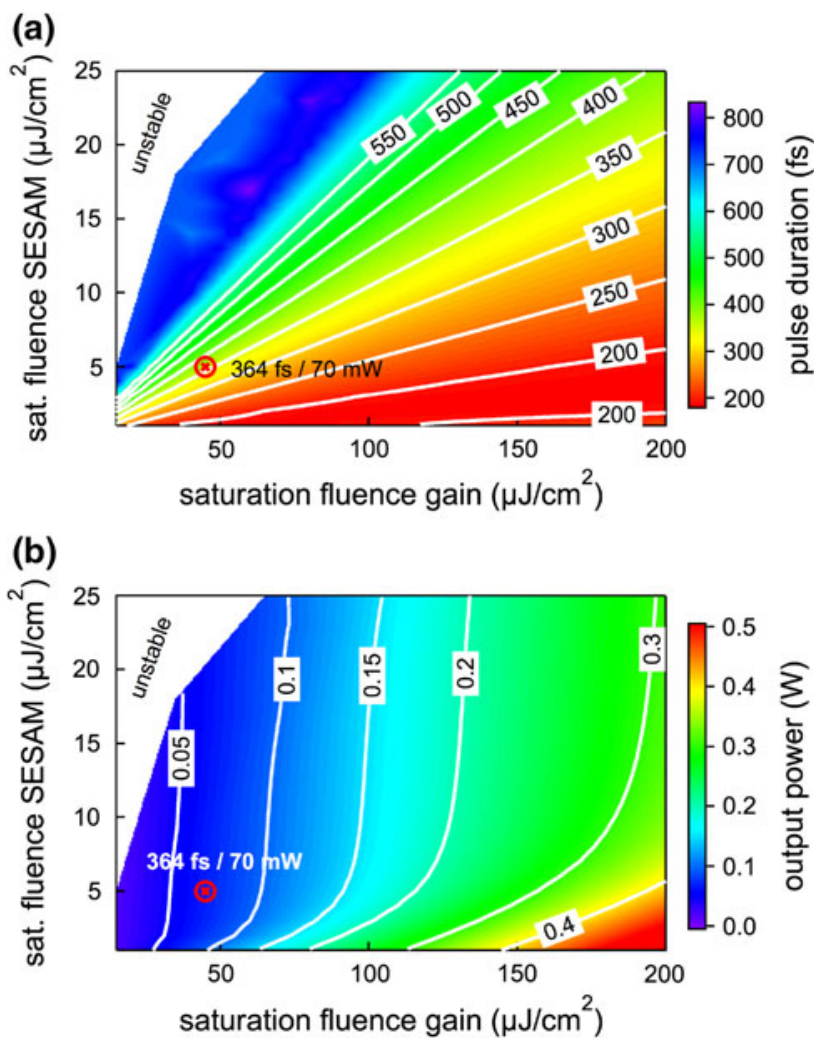

Fig. 12 Influence of the saturation fluence of the gain and the SESAM on $\mathbf{a}$ the pulse duration and $\mathbf{b}$ the average output power. A higher saturation fluence of the gain relaxes the demand for a low saturation fluence regarding short pulse durations, whereas the output power is mainly influenced by the saturation fluence of the gain. The red points on both graphs show the corresponding parameters for the 364-fs result

simulation, we used slightly increased beam spots of $200 \mu \mathrm{m}$ on the VECSEL and the SESAM, which are reasonable sizes and have been already demonstrated in modelocking, for example, in [21]. However, even larger spot sizes of more than $800 \mu \mathrm{m}$ have been used at $\mathrm{cw}$ operation [47], showing that there is still room for further power scaling.

With this design, the model predicts 200 -fs pulses and an average output power of more than $1 \mathrm{~W}$, which at a repetition rate of $2 \mathrm{GHz}$ corresponds to $2.38 \mathrm{~kW}$ peak power. With this pulse duration and peak power, it should be feasible to generate a coherent supercontinuum, thereby enabling the development of VECSEL-based frequency comb technology.

\section{Conclusion}

We present a verified numerical model based on macroscopic measurable parameters. While the SESAM parameters are well-known, recent measurements of important 
Table 4 Parameters for a high-power femtosecond simulation and simulation result

\begin{tabular}{|c|c|c|c|c|c|c|}
\hline Parameter & Gain & Abs. & Cavity parameters & & Results & \\
\hline Saturation fluence $(\mu \mathrm{J} / \mathrm{cm} 2)$ & 100 & 10 & Repetition rate $(\mathrm{GHz})$ & 2.0 & Av. output power (W) & 1.08 \\
\hline Relaxation time $\tau_{g}(\mathrm{~ns})$ & 3 & - & Other losses (\%) & 0.5 & FWHM pulse dur. (fs) & 198 \\
\hline Fast recovery time $\tau_{\text {fast }}(\mathrm{fs})$ & - & 460 & Output coupler $(\%)$ & 1.0 & Peak power $(\mathrm{kW})$ & 2.38 \\
\hline Slow recovery time $\tau_{\text {slow }}(\mathrm{ps})$ & - & 15.6 & Center wavelength (nm) & 960 & FWHM spectrum (nm) & 5.88 \\
\hline Amplitude of slow comp. (\%) & - & 42 & Cavity GDD $\left(\mathrm{fs}^{2}\right)$ & 50 & & \\
\hline Modulation depth $\Delta R(\%)$ & - & 2.0 & Noise floor power (nW) & 10 & & \\
\hline Beam radius $r(\mu \mathrm{m})$ & 200 & 200 & & & & \\
\hline Linewidth enhancement factor $\alpha$ & 3 & 2 & & & & \\
\hline Small-signal gain $g_{\mathrm{ss}}$ & 8 & - & & & & \\
\hline FWHM gain bandwidth (nm) & 30 & - & & & & \\
\hline
\end{tabular}

gain parameters like small-signal gain and saturation fluence almost completed the missing parameters for our model. The recombination time of the gain is assumed to be in the nanosecond range, but ongoing work to measure also this parameter is in progress. In the picosecond regime, a soliton-like pulse formation was experimentally approved, and the femtosecond regime had not been explored and verified yet. Our extensive simulations show that soliton-like pulse shaping is also dominant in this regime and that dispersion management is even more important. We verified this experimentally using a QDVECSEL modelocked by a fast QW-VECSEL, where both devices had a low-dispersion top coating, resulting in an intra-cavity GDD of about $50 \mathrm{fs}^{2}$. We achieved pulse durations as short as $364 \mathrm{fs}$ with an average output power of $70 \mathrm{~mW}$. We obtained excellent agreement with this result and verified also the observed output power for the first time. Furthermore, a recent experiment, where the repetition rate of a modelocked femtosecond VECSEL was tuned continuously over a wide range of repetition rates, corresponding to significant pulse energy changes, was numerically reproduced. These results quantitatively verify our numerical model in the femtosecond regime and also confirm the soliton-like pulse shaping.

With these results, we have a powerful tool to provide a guideline for new VECSEL and SESAM designs to get few 100-fs pulses with output powers in the Watt level (in 1:1 modelocking). We show that for the gain, it is important to increase the saturation fluence and the small-signal gain to achieve higher output powers and shorter pulses. For the SESAM, it is crucial to have low saturation fluence combined with a relatively high modulation depth, to get short pulses. Taking all of these considerations into account, we designed a VECSEL which, based on our model, should yield 200-fs pulses and an average output power exceeding $1 \mathrm{~W}$.

Acknowledgments We would like to thank Dr. Christopher Phillips for helpful discussions. This work was supported by ETH Zrich with the FIRST clean room facility and was financed by the Swiss Confederation Program Nano-Tera.ch, which was scientifically evaluated by the SNSF and by the European Community's Seventh Framework Program FAST-DOT under Grant agreement 224338. The authors also wish to acknowledge the work of the open source community: Our numerical model makes use of the following open source projects and libraries: (1) Python (http://www.python.org), (2) SciPy (http:// www.scipy.org), (3) NumPy (http://www.numpy.org), (4) ParallelPython (http://www.parallelpython.org)

Open Access This article is distributed under the terms of the Creative Commons Attribution License which permits any use, distribution, and reproduction in any medium, provided the original author(s) and the source are credited.

\section{References}

1. M. Kuznetsov, F. Hakimi, R. Sprague, A. Mooradian, IEEE Photonics Technol. Lett. 9, 1063 (1997)

2. U. Keller, A.C. Tropper, Phys. Rep. Rev. Sect. Phys. Lett. 429, 67 (2006)

3. U. Keller, K.J. Weingarten, F.X. Kärtner, D. Kopf, B. Braun, I.D. JUNG, R. Fluck, C. Honninger, N. Matuschek, J. Ausder Au, IEEE J. Sel. Top. Quantum Electron. 2, 435 (1996)

4. U. Keller, D.A.B. Miller, G.D. Boyd, T.H. Chiu, J.F. Ferguson, M.T. Asom, Opt. Lett. 17, 505 (1992)

5. U. Keller, Appl. Phys. B 100, 15 (2010)

6. D.J.H.C. Maas, A.-R. Bellancourt, B. Rudin, M. Golling, H.J. Unold, T. Südmeyer, U. Keller, Appl. Phys. B 88, 493 (2007)

7. A.-R. Bellancourt, D.J.H.C. Maas, B. Rudin, M. Golling, T. Südmeyer, U. Keller, IET Optoelectron. 3, 61 (2009)

8. C. Honninger, R. Paschotta, F. Morier-Genoud, M. Moser, U. Keller, J. Opt. Soc. Am. B Opt. Phys. 16, 46 (1999)

9. S. Calvez, J.E. Hastie, M. Guina, O.G. Okhotnikov, M.D. Dawson, Laser Photonics Rev. 3, 407 (2009)

10. V.J. Wittwer, C.A. Zaugg, W.P. Pallmann, A.E.H. Oehler, B. Rudin, M. Hoffmann, M. Golling, Y. Barbarin, T. Südmeyer, U. Keller, Electron. Lett. 3, 658 (2011)

11. K.G. Wilcox, H.D. Foreman, J.S. Roberts, A.C. Tropper, Electron. Lett. 42, 159 (2006)

12. A. Schlatter, B. Rudin, S.C. Zeller, R. Paschotta, G.J. Spuhler, L. Krainer, N. Haverkamp, H.R. Telle, U. Keller, Opt. Lett. 30, 1536 (2005)

13. D. Hillerkuss, T. Schellinger, R. Schmogrow, M. Winter, T. Vallaitis, R. Bonk, A. Marculescu, J. Li, M. Dreschmann, J. 
Meyer, S. Ben Ezra, N. Narkiss, B. Nebendahl, F. Parmigiani, P. Petropoulos, B. Resan, K. J. Weingarten, T. Ellermeyer, J. Lutz, M. Möller, M. Huebner, J. Becker, C. Koos, W. Freude, J. Leuthold, in Optical Fiber Communication Conference (OFC) (San Diego, USA, 2010), post-deadline talk PDPC1

14. M.C. Stumpf, S. Pekarek, A.E.H. Oehler, T. Südmeyer, J.M. Dudley, U. Keller, Appl. Phys. B 99, 401 (2009)

15. S. Schilt, N. Bucalovic, V. Dolgovskiy, C. Schori, M.C. Stumpf, G.D. Domenico, S. Pekarek, A.E.H. Oehler, T. Südmeyer, U. Keller, P. Thomann, Opt. Express 19, 24171 (2011)

16. H.R. Telle, G. Steinmeyer, A.E. Dunlop, J. Stenger, D.H. Sutter, U. Keller, Appl. Phys. B 69, 327 (1999)

17. M.J.W. Rodwell, D.M. Bloom, K.J. Weingarten, IEEE J. Quantum Electron. 25, 817 (1989)

18. S. Schilt, V. Dolgovskiy, N. Bucalovic, C. Schori, M. C. Stumpf, G. Domenico, S. Pekarek, A. E. H. Oehler, T. Südmeyer, U. Keller, P. Thomann, Applied Physics B, 109, 391 (2012)

19. S. Pekarek, T. Südmeyer, S. Lecomte, S. Kundermann, J.M. Dudley, U. Keller, Opt. Express 19, 16491 (2011)

20. S. Pekarek, C. Fiebig, M.C. Stumpf, A.E.H. Oehler, K. Paschke, G. Erbert, T. Südmeyer, U. Keller, Opt. Express 18, 16320 (2010)

21. B. Rudin, V.J. Wittwer, D.J.H.C. Maas, M. Hoffmann, O.D. Sieber, Y. Barbarin, M. Golling, T. Südmeyer, U. Keller, Opt. Express 18, 27582 (2010)

22. M. Hoffmann, O.D. Sieber, V.J. Wittwer, I.L. Krestnikov, D.A. Livshits, Y. Barbarin, T. Südmeyer, U. Keller, Opt. Express 19, 8108 (2011)

23. S. Chen, K.J. Schafer, M.B. Gaarde, Opt. Letters 37, 2211 (2012)

24. P. Klopp, U. Griebner, M. Zorn, M. Weyers, Appl. Phys. Lett. 98, 071103 (2011)

25. K.G. Wilcox, V. Apostolopoulos, Z. Mihoubi, S.P. Elsmere, I. Farrer, D.A. Ritchie, A. Tropper, Nat. Photonics 3, 729 (2009)

26. R. Paschotta, R. Häring, A. Garnache, S. Hoogland, A. Tropper, U. Keller, Appl. Phys. B 75, 445 (2002)

27. M. Hoffmann, O.D. Sieber, D.J.H.C. Maas, V.J. Wittwer, M. Golling, T. Südmeyer, U. Keller, Opt. Express 18, 10143 (2010)

28. M. Mangold, V.J. Wittwer, O.D. Sieber, M. Hoffmann, I.L. Krestnikov, D.A. Livshits, M. Golling, T. Südmeyer, U. Keller, Opt. Express 20, 4136 (2012)
29. D.J.H.C. Maas, B. Rudin, A.-R. Bellancourt, D. Iwaniuk, S.V. Marchese, T. Südmeyer, U. Keller, Opt. Express 16, 7571 (2008)

30. M. Haiml, R. Grange, U. Keller, Appl. Phys. B-Lasers Opt. 79, 331 (2004)

31. O.D. Sieber, V.J. Wittwer, M. Mangold, M. Hoffmann, M. Golling, T. Südmeyer, U. Keller, Opt. Express 19, 23538 (2011)

32. R. Paschotta, Appl. Phys. B 79, 153 (2004)

33. G.H.C. New, Opt. Commun. 6, 188 (1972)

34. O.E. Martinez, R.L. Fork, J.P. Gordon, J. Opt. Soc. Am. B Opt. Phys. 2, 753 (1985)

35. C.H. Henry, IEEE J. Quantum Electron. 18, 259 (1982)

36. M.E. Barnes, Z. Mihoubi, K.G. Wilcox, A.H. Quarterman, I. Farrer, D.A. Ritchie, A. Garnache, S. Hoogland, V. Apostolopoulos, A.C. Tropper, Opt. Express 18, 21330 (2010)

37. D.J.H.C. Maas, A.-R. Bellancourt, M. Hoffmann, B. Rudin, Y. Barbarin, M. Golling, T. Südmeyer, U. Keller, Opt. Express 16, 18646 (2008)

38. K. Naganuma, K. Mogi, H. Yamada, Opt. Lett. 15, 393 (1990)

39. A. Gosteva, M. Haiml, R. Paschotta, U. Keller, J. Opt. Soc. Am. B Opt. Phys. 22, 1868 (2005)

40. W. Jiang, J. Bowers, Compact Sources of Ultrashort Pulses, (Cambridge University Press, New York, 1995) pp. 208-273.

41. F. Kartner, I. Jung, U. Keller, IEEE J. Sel. Top. Quantum Electron. 2, 540 (1996)

42. I.D. Jung, F.X. Kärtner, L.R. Brovelli, M. Kamp, U. Keller, Opt. Lett. 20, 1892 (1995)

43. S. Melnik, G. Huyet, A. Uskov, Opt. Express 14, 2950 (2006)

44. G.P. Agrawal, C.M. Bowden, IEEE Photonics Technol. Lett. 5, 640 (1993)

45. R. Grange, M. Haiml, R. Paschotta, G.J. Spuhler, L. Krainer, M. Golling, O. Ostinelli, U. Keller, Appl. Phys. B 80, 151 (2004)

46. R. Paschotta, U. Keller, Appl. Phys. B 73, 653 (2001)

47. B. Heinen, T.L. Wang, M. Sparenberg, A. Weber, B. Kunert, J. Hader, S.W. Koch, J.V. Moloney, M. Koch, W. Stolz, Electron. Lett. 48, 516 (2012) 